\title{
' $\mathbf{C}_{60}$ Lunar Lander' Constructs Direct Electrons at Electrode Interfaces
}

\section{Key words}

fullerenes

nanoscience

electrocatalysis

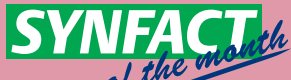

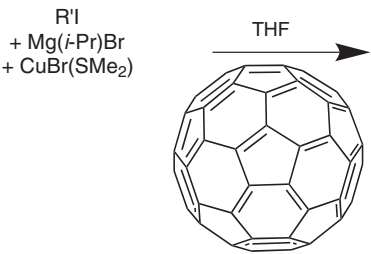

$R^{\prime}=$ ethyl ester of $R$

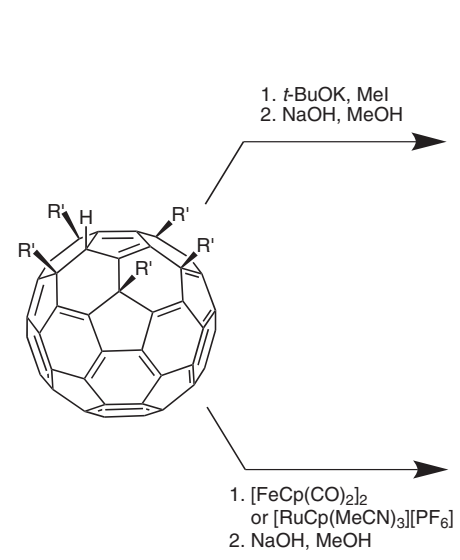

photo-reduction
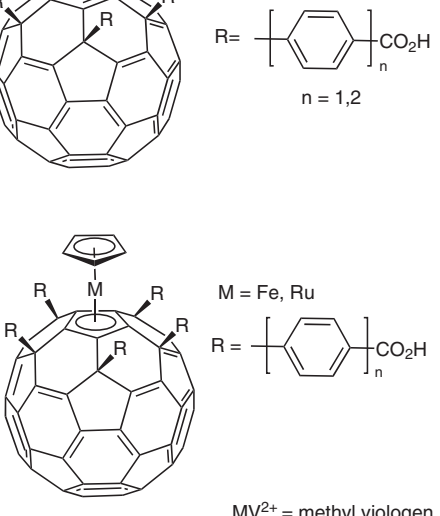

AsA = ascorbic acid
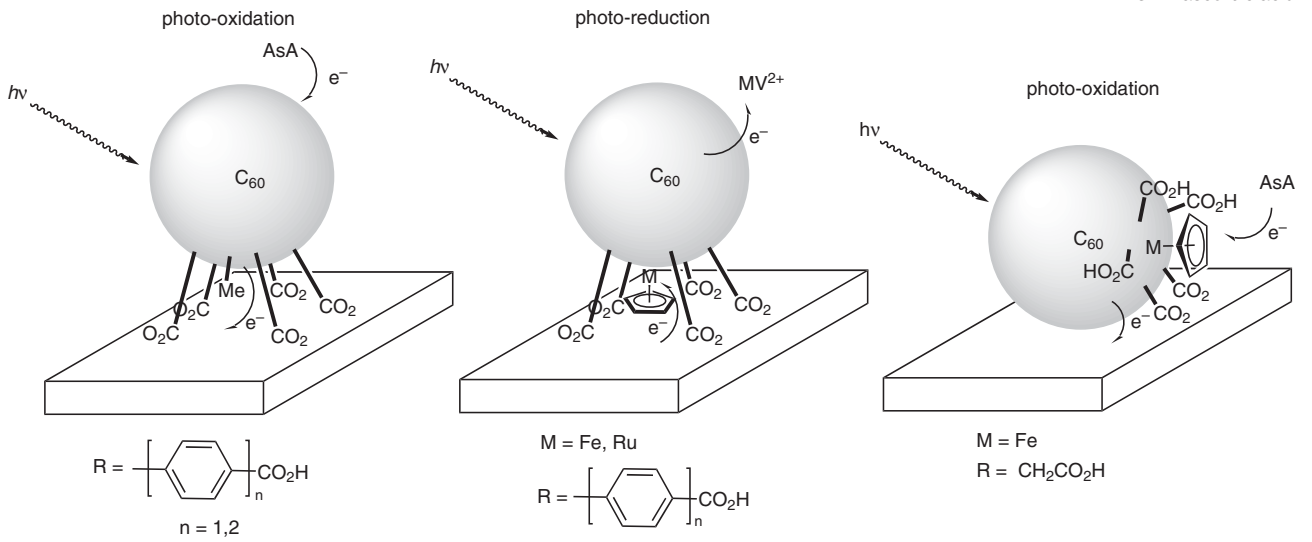

Significance: Functionalized fullerenes bound to indium tin oxide electrodes create photocurrents with illumination. When all of the carboxylates bind the surface the fullerenes display either photo-oxidation or photo-reduction behavior depending upon the functionalization. In the case of the organometallic complexes, initial irradiation of the fullerene results in rapid oxidation of the metal center and hence the fullerene is not able to oxidize AsA but is able to reduce $\mathrm{MV}^{2+}$. With short legs $\left(\mathrm{R}=\mathrm{CH}_{2} \mathrm{CO}_{2} \mathrm{H}\right)$ the complex must lay on its side and the metal center is capable of reacting with the AsA.
Comment: The synthetic methods used in this study are well established, however, the ability to control the direction of electron flow at the surfaces by design is very novel. This work demonstrates that well-defined structurally rigid platforms can give precise direction to the nature of oxidation or reduction at surfaces. Similar designs can be used for creating precise electrocatalytic systems and molecular electronic devices.

SYNFACTS Contributors: Timothy M. Swager

Synfacts 2008, 7, 0703-0703 Published online: 20.06.2008 Dol: 10.1055/s-2008-1077811; Reg-No.: S05408SF 